\section{Enfermedad de Chagas en Argentina: herramientas para que los escolares vigilen y determinen la presencia de factores de riesgo en sus viviendas}

\author{
Chagas disease in Argentina: \\ tools for schoolchildren to exercise vector \\ surveillance and identify household risk factors
}

\author{
Liliana Crocco 1 \\ Claudia Rodríguez 1 \\ Silvia Catalá 2 \\ Julieta Nattero ${ }^{1}$
}

\footnotetext{
1 Facultad de Ciencias Exactas, Físicas y Naturales, Universidad Nacional de Córdoba, Córdoba, Argentina.

2 Centro Regional de Investigaciones Científicas y Transferencia Tecnológica, La Rioja, Argentina.

Correspondencia L. Crocco

Centro de Investigaciones Entomológicas, Facultad de Ciencias Exactas,

Físicas y Naturales, Universidad de Córdoba. Vélez Sársfield 299, (X5000JJC),

Córdoba, Argentina. lcrocco@com.uncor.edu
}

\begin{abstract}
The objective of this paper was to validate tools for schoolchildren to help control Chagas disease vectors in their own homes. Two types of tables were produced, one reporting on the presence of risk factors in residences and the other to record the presence of triatomines. The materials were tested in the field by 100 students from an endemic area in Argentina and validated by technical personnel. The results obtained by students concerning the percentage of households with risk factors and vector-positive residences were similar to those obtained by the technical personnel. This information allowed teachers to classify the risk levels in the residences as at "high-risk", "risk", or "no risk". The information provides an important aid for health workers, and it is therefore recommended to include this educational strategy in the surveillance phase of the Chagas disease control program.
\end{abstract}

Chagas Disease; Risk Factors; Vector Control

\section{Introducción}

Los programas de control vectorial en Argentina han logrado un fuerte impacto en la disminución de la transmisión del Trypanosoma cruzi por la vía vectorial 1 . No obstante, los programas de participación comunitaria para la etapa de vigilancia actualmente están limitados y en varias regiones ausentes. Esto debido entre otros factores a necesidades básicas insatisfechas y preocupaciones por la fuente de trabajo. Además, como se trata de una enfermedad cuyos efectos o sintomatología no son observados inmediatamente, se hace más difícil la participación de la comunidad en acciones de control y vigilancia, más aún en época de fuerte crisis social y económica 2 . Considerando estas problemáticas y planteada la necesidad de una vigilancia sostenida en el tiempo ${ }^{3}$, se propone a la escuela como el ámbito más propicio para brindar a los escolares de áreas endémicas la información y las herramientas necesarias para emprender acciones directas que lleven a la disminución del riesgo de contraer la enfermedad de Chagas.

En este marco el objetivo de este trabajo es validar en campo herramientas que permitan desde la escuela vigilar las viviendas y determinar la presencia de factores de riesgo. 


\section{Metodología}

El trabajo se desarrolló durante los años 20012002 en áreas rurales de los departamentos de Castro Barros y Río Seco de las provincias de La Rioja y Córdoba respectivamente, en Argentina, donde todavía se registra transmisión vectorial activa por la especie Triatoma infestans (especie domiciliaria y peridomiciliaria). Los porcentajes de infestación intradomicilar y peridomiciliar para Córdoba en el año 2002 fueron del $2,50 \%$ y $11,43 \%$ respectivamente, para La Rioja $5,47 \%$ y $8,96 \%$ (Comisión Intergubernamental de los Países del Cono Sur para la Eliminación de T. infestans. Informe técnico. Presentado en la XIIa Reunión de la Comisión Intergubernamental del Cono Sur para la Eliminación de Triatoma infestans y la Interrupción de la Transmisión Transfusional de la Trypanosomiasis Americana (INCOSUR/Chagas); 2003).

Se trabajó con 100 alumnos de los últimos grados del nivel primario, pertenecientes a escuelas rurales de ambos departamentos. La selección de escuelas y viviendas se realizó en coordinación con los Ministerios de Educación y el Servicio Nacional de Chagas, teniendo como criterio áreas no tratadas en los últimos años y ausencia de actividades educativas y de participación comunitaria.

\section{Características de las planillas}

Se utilizaron dos planillas, una para caracterizar la vivienda basada en la presencia de factores de riesgo que posibilitan el refugio de las vinchucas dentro y fuera de la vivienda y que son fácilmente detectables por los niños 4. A cada factor se le asignó un puntaje 5,6,7,8,9, la sumatoria de estos puntos permitió obtener el grado de riesgo de la vivienda conforme se presenta en la Tabla 1 . Se consideró como "con mucho riesgo" entre 85 y 34 puntos; "de riesgo" entre 33 y 13 puntos y vivienda "sin riesgo" cuando se obtiene menos de 13 puntos.

La otra planilla para la vigilancia entomológica 5 fue diseñada para que el alumno registre la presencia o ausencia de vinchucas y/o rastros (heces, muda, huevos), tanto dentro de la vivienda como en sus alrededores.

\section{Puesta a prueba en campo y validación}

La prueba en campo la realizaron los escolares entrenados por sus docentes que previamente
Tabla 1

Factores de riesgos incluidos en las planillas de los escolares: categorías y valores asignados a cada uno para obtener nivel de riesgo de las viviendas.

\begin{tabular}{|c|c|}
\hline Factor de riesgo & Valor asignado \\
\hline \multicolumn{2}{|l|}{ Tipo de vivienda } \\
\hline \multicolumn{2}{|l|}{ Techo } \\
\hline Paja y barro & 14 \\
\hline Caña y varillas & 12 \\
\hline Chapa y paja & 10 \\
\hline Chapas solas & 5 \\
\hline Material & 1 \\
\hline Otros & 0 \\
\hline \multicolumn{2}{|l|}{ Paredes } \\
\hline Adobe & 14 \\
\hline Ladrillos sin revoque & 10 \\
\hline Piedras & 8 \\
\hline Ladrillos con revoque & 3 \\
\hline Otro material & 0 \\
\hline \multicolumn{2}{|l|}{ Grietas en las paredes } \\
\hline Sí & 4 \\
\hline No & 0 \\
\hline \multicolumn{2}{|c|}{$\begin{array}{l}\text { Cajas/ropa amontonada en el interior de la vivienda } \\
\text { (desorden en la vivienda) }\end{array}$} \\
\hline Sí & 8 \\
\hline \multicolumn{2}{|c|}{ Distancia del gallinero a la vivienda (en pies) } \\
\hline Entre 0 y 20 & 12 \\
\hline Entre 20 y 40 & 10 \\
\hline Entre 40 y 60 & 8 \\
\hline Más de 60 & 3 \\
\hline \multicolumn{2}{|c|}{ Distancia del corral a la vivienda (en pies) } \\
\hline Entre 0 y 20 & 9 \\
\hline Entre 20 y 40 & 7 \\
\hline Entre 40 y 60 & 5 \\
\hline Más de 60 & 2 \\
\hline \multicolumn{2}{|c|}{ Distancia depósito de la vivienda (en pies) } \\
\hline Entre 0 y 20 & 4 \\
\hline Entre 20 y 40 & 3 \\
\hline Entre 40 y 60 & 2 \\
\hline Más de 60 & 0 \\
\hline
\end{tabular}


fueron capacitados a través de un módulo de actualización en Chagas 10 y otro con sugerencias para trabajar en el aula 5. Los escolares aplicaron las planillas en sus viviendas y en caso de encontrar vinchucas las recolectaron en frascos de acuerdo a indicaciones del docente. A partir de la información recabada, los docentes obtuvieron el grado de riesgo de las viviendas, información que luego se comunica al centro de salud más próximo.

La validación de las planillas y el trabajo de los escolares la realizó un equipo técnico que aplicó las mismas planillas en 50 viviendas que se seleccionaron al azar de entre las evaluadas por los escolares. Luego se compararon estadísticamente (diferencia de porcentajes) los valores obtenidos para cada ítem de las planillas con los obtenidos por los escolares.

\section{Análisis estadístico}

Los datos se registraron y analizaron mediante el programa estadístico SPSS 10.0. Además de estadísticos descriptivos, se utilizó la prueba de chi-cuadrado con corrección de Yates, aplicada a tablas de contingencia.

\section{Resultados}

Las condiciones para que un escolar pueda actuar como vigilante de las viviendas se presentan en la Tabla 2.

\section{Características de las viviendas: presencia de factores de riesgos}

Los porcentajes de viviendas que presentan factores de riesgos evaluadas por los escolares no mostraron diferencias significativas con los porcentajes obtenidos por el personal técnico,

Tabla 2

Característica del alumno vigilante de su vivienda.

Condiciones para ser alumno vigilante

Tener conocimientos básicos sobre la enfermedad de Chagas

Reconocer a las vinchucas en sus distintos estadios: huevo, ninfa, adulto

Identificar las heces de las vinchucas

Reconocer cuáles son los posibles refugios de las vinchucas

Identificar dentro y fuera de su vivienda la presencia o indicios de vinchucas

e informar a la escuela a través de la planilla de seguimiento entomológico

Identificar los principales factores de riesgo en la vivienda y peridomicilio

e informar a la escuela a través del registro en planillas excepto el factor desorden en la vivienda (Tabla 3). En ambos departamentos los escolares registraron un porcentaje significativamente menor de viviendas con desorden en su interior. En relación al nivel de riesgo, el departamento de Río Seco presentó un porcentaje significativamente mayor $(45,50 \%)$ de viviendas con mucho riesgo respecto al departamento de Castro Barros (23,70\%) (Figura 1).

Presencia de vinchucas y/o rastros

Del análisis de las planillas de vigilancia entomológica se obtuvieron los porcentajes de viviendas con presencia de vinchucas y/o rastros dentro de las viviendas y/o en su peridomicilio (Tabla 4). No se registraron diferencias significativas entre la evaluación realizada por los escolares con la del personal técnico excepto en el departamento de Castro Barros, donde el registro de los escolares fue significativamente menor que la de los técnicos. El departamento de Río Seco presentó los porcentajes significativamente más altos de viviendas con presencia de vinchucas y/o rastros en los peridomicilios.

Los análisis de chi-cuadrado, coeficiente de contingencia, phi y $v$ de Cramer y lambda permitieron registrar dependencia entre las variables tipo de vivienda y presencia de vinchucas y una asociación moderadamente fuerte entre ambas (nivel de significación de 0,0001).

\section{Discusión}

Los resultados obtenidos en este trabajo demuestran que a través de herramientas sencillas los escolares previamente entrenados, no sólo pueden participar activamente en las tareas de vigilancia de la enfermedad de Chagas, sino también caracterizar las viviendas en grados de riesgos.

En cuanto a la validez de las planillas, los registros de los escolares coincidieron con la del personal técnico y con los registrados por Sanmartino \& Crocco ${ }^{4}$. El factor "vivienda en desorden” fue el que mostró diferencias, lo cual se sugiere que habría que contextualizar este factor teniendo en cuenta las percepciones y cultura propia de cada región.

A pesar de esta dificultad, los resultados obtenidos estarían indicando que es factible que los escolares puedan vigilar las viviendas aplicando estas planillas. Por otra parte, la información que puede obtener la escuela sobre nivel de riesgo de las viviendas puede ser una importante ayuda para los agentes primarios de 
Porcentaje de viviendas evaluadas por escolares y técnicos, que presentan factores de riesgo en los departamentos de Río Seco y Castro Barros, Argentina.

\begin{tabular}{|c|c|c|c|c|}
\hline \multirow[t]{2}{*}{ Factores de riesgo } & \multicolumn{2}{|c|}{$\begin{array}{l}\text { Departamento de } \\
\text { Río Seco (\%) }\end{array}$} & \multicolumn{2}{|c|}{$\begin{array}{l}\text { Departamento de } \\
\text { Castro Barros (\%) }\end{array}$} \\
\hline & escolares & técnicos & escolares & técnicos \\
\hline Techo con riesgo & 77,2 & 71,4 & 34,2 & 38,5 \\
\hline Pared con riesgo & 54,6 & 78,4 & 34,3 & 46,2 \\
\hline Presencia de grietas en las paredes & 43,3 & 64,3 & 39,2 & 46,4 \\
\hline Desorden en la vivienda & $20,0 a$ & $64,3 b$ & $13,9 c$ & $40,0 d$ \\
\hline Gallineros próximos a las viviendas & 35,0 & 50,0 & $13,6 \mathrm{e}$ & $36,0 f$ \\
\hline Corrales próximos a las viviendas & 5,1 & 1,0 & $17,6 \mathrm{~g}$ & $33,0 h$ \\
\hline Perros dentro de las viviendas & 9,1 & 23,0 & 23,6 & 10,0 \\
\hline Aves dentro de las viviendas & 19,0 & 28,0 & 16,3 & 12,5 \\
\hline Total de viviendas & 24,0 & 18,0 & 76,0 & 32,0 \\
\hline
\end{tabular}

a y $b=$ difieren significativamente entre sí a $p<0,005$; c y $d=$ difieren significativamente entre sí a $p<0,005$;

e y $f=$ difieren significativamente entre sí a $p<0,005$; g y $=$ difieren significativamente entre sí a $p<0,001$.

Figura 1

Porcentajes de viviendas con mucho riesgo, de riesgo y sin riesgo en los departamentos de Río Seco

y Castro Barros. Argentina, 2001-2002.

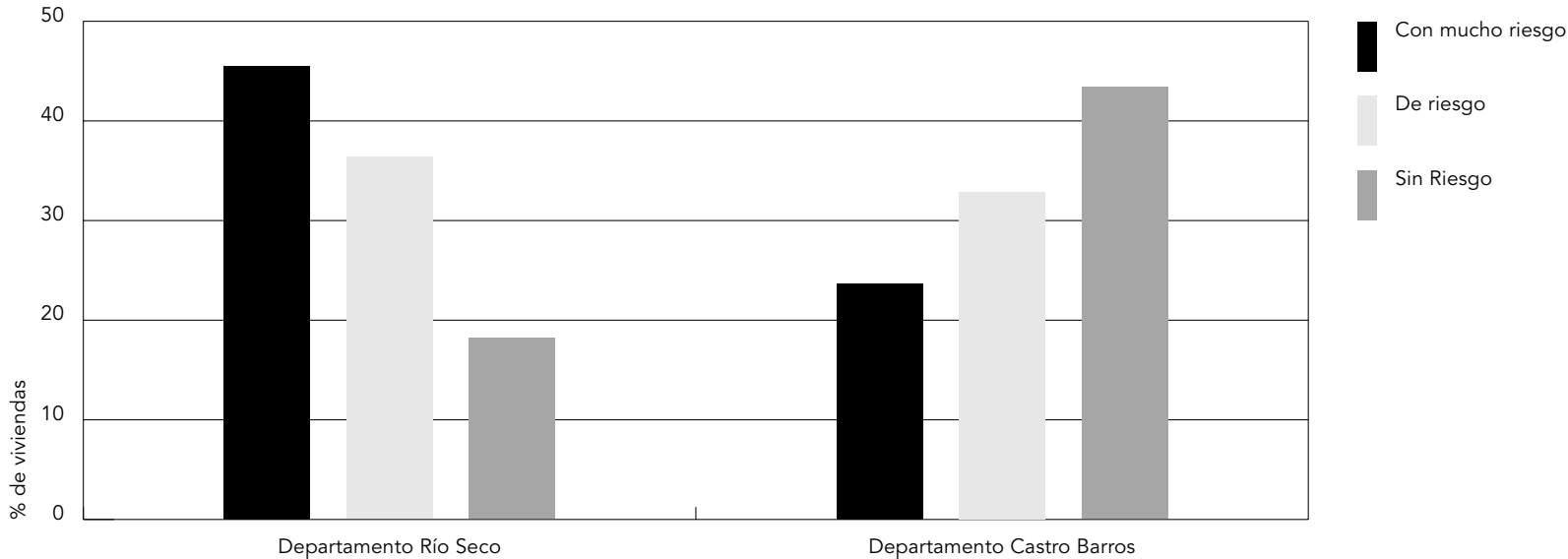

salud, ya que dispondrán de un pequeño mapa local de riesgo donde podrán definir qué viviendas tienen que vigilar con mayor asiduidad, optimizando tiempos y esfuerzos. El seguimiento de las viviendas por los escolares podría ser mensual o de no ser posible de acuerdo al área endémica, seleccionar aquellos meses de mayor riesgo de transmisión. Por ejemplo, para Argentina se propone una vez al mes, desde agosto hasta finalizar las clases (noviembre-diciembre), ya que estos serían los meses de mayor riesgo de transmisión 11.

Para que esta propuesta sea efectiva se requiere establecer acuerdos entre el área de salud y de educación que garanticen la conexión escuela-salud-comunidad. También se recomienda que se implementen programas de capacitación docente continua, como también 
Porcentaje de viviendas evaluadas por escolares y técnicos, que presentan vinchucas y/o rastros en la viviendas y en sus peridomicilios en los departamentos de Río Seco y Castro Barros (Argentina).

\begin{tabular}{|c|c|c|c|c|}
\hline \multirow[t]{2}{*}{ Presencia de vinchucas $y / 0$ rastros } & \multicolumn{2}{|c|}{$\begin{array}{l}\text { Departamento de Río } \\
\text { Seco (Córdoba) (\%) }\end{array}$} & \multicolumn{2}{|c|}{$\begin{array}{c}\text { Departamento de Castro } \\
\text { Barros (La Rioja) (\%) }\end{array}$} \\
\hline & escolares & técnicos & escolares & técnicos \\
\hline Presencia dentro de la vivienda & 18,2 & 21,4 & 10,5 & 8,3 \\
\hline Presencia en los peridomicilios & 31,8 & 42,9 & $13,2 \mathrm{a}$ & $33,3 b$ \\
\hline Total de viviendas & 24,0 & 18,0 & 76,0 & 32,0 \\
\hline
\end{tabular}

a y $b=$ difieren significativamente entre sí $a p<0,005$.

que la escuela disponga de material informativo actualizado 5,10 .

La importancia de incorporar esta estrategia educativa como medida complementaria a las acciones de vigilancia, no sólo será impor- tante en la reducción de costos, sino que se puede disponer de información de áreas con riesgos durante todo el año, lo cual permitiría optimizar los tiempos y la evaluación del personal técnico.

\section{Resumen}

El objetivo de este trabajo fue validar herramientas para que escolares puedan vigilar sus viviendas. Se desarrollaron dos tipos de planillas, una sobre la presencia de factores de riesgos y otra para registrar la presencia de vinchucas en las viviendas. Estas planillas fueron puestas a prueba en campo por 100 escolares de escuelas de áreas endémicas de Argentina y validadas en campo por personal técnico. Los resultados obtenidos por los escolares sobre porcentajes de viviendas que presentan factores de riesgos y viviendas positivas fueron similares a los obtenidos por el personal técnico. La información recabada permitió a los docentes caracterizar las viviendas como "con mucho riesgo", "de riesgo" o "sin riesgo". Esta información sobre nivel de riesgo de las viviendas puede ser una importante ayuda para los agentes primarios de salud. Por lo tanto, se recomienda incluir estas estrategias educativas en los programas de control, en especial para la fase de vigilancia.

Enfermedad de Chagas; Factor de Riesgo; Control Vectorial

\section{Colaboradores}

L. Crocco participó en todas las instancias de desarrollo del trabajo en campo, análisis de datos y escritura del artículo. C. Rodríguez participó en el diseño de las estrategias, validación en campo, análisis de datos, discusión final. S. Catalá participó fundamentalmente en trabajo de campo, escritura y discusión de resultados. J. Nattero participó en el diseño de las estrategias, validación en campo, análisis de datos, discusión final.

\section{Agradecimientos}

Al Servicio Nacional de Chagas y a los Ministerios de Educación de Córdoba y La Rioja, especialmente a docentes y alumnos de las escuelas rurales de Río Seco y Castro Barros y habitantes en general. Agradecemos también la valiosa colaboración con las encuestas de Ana López y Cecilia Estrabou. El desarrollo de este trabajo se realizó con subsidio otorgado por el Fondo para la Investigación Científica, Argentina (FONCYT, PICT 99, 05 - 07077). 


\section{Referencias}

1. Blanco S. Programa nacional de control de la Enfermedad de Chagas en Argentina. 2o Simposio Internacional de Enfermedad de Chagas en Internet. http://www.fac.org.ar/fec/chagas2/marcos/ marcos.htm (accesado el 25/Nov/2003).

2. Salomón D. Aspectos de prevención de la enfermedad de Chagas con participación de la comunidad. 2o Simposio Internacional de Enfermedad de Chagas en Internet. http://www.fac.org.ar/ fec/chagas $2 /$ marcos/marcos.htm (accesado el 25/Nov/2003).

3. Pinto Días JC. Problemas e possibilidades de participação comunitária no controle das grandes endemias no Brasil Cad Saúde Pública 1998; 14 Suppl 2:19-37.

4. Sanmartino M, Crocco L. Conocimientos sobre la enfermedad de Chagas y factores de riesgo en comunidades epidemiológicamente diferentes de Argentina. Rev Panam Salud Pública 2000; 7:173-8.

5. Crocco L, De Longhi A, Catalá S. Enfermedad de Chagas: sugerencias para trabajar en el aula. Córdoba: Editorial Universitas; 2002.

6. Cecere MC, Gürtler RE, Canale D, Chuit R, Cohen JE. El papel del peridomicilio en la eliminación de Triatoma infestans de comunidades rurales argentinas. Bol Oficina Sanit Panam 1996; 121:1-8.
7. Gürtler R, Cecere MC, Petersen RM, Rubel DN Schweigmann N. Chagas disease in north-west Argentina: association between Trypanosoma cruzi parasitemia in dogs and cats and infection rates in domestic Triatoma infestans. Trans R Soc Trop Med Hyg 1993; 87:12-5.

8. Lopez A, Crocco L, Morales G, Catalá S. Chicken nest as risk factors for Chagas disease. Mem Inst Oswaldo Cruz 1997; 92 Suppl 1:92.

9. Paulone I. Factores socio-económicos, culturales y ecológicos asociados a reinfestaciones por Triatoma infestans en viviendas rurales del área de Santiago del Estero, bajo vigilancia epidemiológica [PhD Thesis]. Santa Fe: Universidad del Litoral; 1994.

10. Crocco L, Catalá S, Martínez M. Enfermedad de Chagas: módulo de actualización. Córdoba: Editorial Universitas; 2002.

11. Catalá S, Crocco L, Morales G, Paulone I, Giraldez E, Candiotti C, et al. Preliminary outlook of Chagas disease transmission risk on different geographic areas of Argentina. Mem Inst Oswaldo Cruz 1996; 91 Suppl:72.

Recibido el 18/Jun/2004

Versión final presentada el 20/Oct/2004

Aprobado el 19/Nov/2004 